\title{
CLASSIFICATION OF DEGREE 2 POLYNOMIAL AUTOMORPHISMS OF $\mathbb{C}^{3}$
}

\author{
John ERIK Fornæss And He Wu
}

\begin{abstract}
For the family of degree at most 2 polynomial self-maps of $\mathbb{C}^{3}$ with nowhere vanishing Jacobian determinant, we give the following classification: for any such map $f$, it is affinely conjugate to one of the following maps:

(i) An affine automorphism;

(ii) An elementary polynomial autormorphism

$$
E(x, y, z)=(P(y, z)+a x, Q(z)+b y, c z+d),
$$

where $P$ and $Q$ are polynomials with $\max \{\operatorname{deg}(P), \operatorname{deg}(Q)\}=2$ and $a b c \neq 0$.

(iii)

$$
\left\{\begin{array}{l}
H_{1}(x, y, z)=(P(x, z)+a y, Q(z)+x, c z+d) \\
H_{2}(x, y, z)=(P(y, z)+a x, Q(y)+b z, y) \\
H_{3}(x, y, z)=(P(x, z)+a y, Q(x)+z, x) \\
H_{4}(x, y, z)=(P(x, y)+a z, Q(y)+x, y) \\
H_{5}(x, y, z)=(P(x, y)+a z, Q(x)+b y, x)
\end{array}\right.
$$

where $P$ and $Q$ are polynomials with $\max \{\operatorname{deg}(P), \operatorname{deg}(Q)\}=2$ and $a b c \neq 0$.
\end{abstract}

\section{Introduction}

In this note, we will give a classification theorem for the family of degree at most 2 polynomial self-maps of $\mathbb{C}^{3}$ with nowhere vanishing Jacobian determinant. Note that any polynomial automorphism has a nowhere vanishing Jacobian determinant. Our Theorem 2.1 implies that any degree at most 2 polynomial self-map of $\mathbb{C}^{3}$ with nowhere vanishing Jacobian determinant is a polynomial automorphism. 
Let $\mathcal{G}$ be the group of polynomial automorphisms of $\mathbb{C}^{2}$. Let $\mathcal{A}$ be the group of affine automorphisms of $\mathbb{C}^{2}$ and let $\mathcal{E}$ be the group of elementary polynomial automorphisms of $\mathbb{C}^{2}$ such that each $e \in \mathcal{E}$ is of the form:

$$
e(x, y)=(a x+P(y), b y+c)
$$

where $P$ is a polynomial and $a, b \neq 0$. Note that $\mathcal{E}$ is the group of all polynomial automorphisms that carry each line of the form $y=c o n$ -

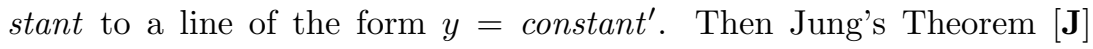
asserts that $\mathcal{G}$ is generated by $\mathcal{A}$ and $\mathcal{E}$. Applying Jung's Theorem, Friedland and Milnor $[\mathbf{F M}]$ classified the polynomial automorphisms of $\mathbb{C}^{2}$ : Any polynomial automorphism of $\mathbb{C}^{2}$ is affinely conjugate to one of the following types of maps: (i) an affine automorphism; (ii) an elementary polynomial automorphism; (iii) A finite composition of generalized Hénon mappings. Each generalized Hénon mapping is of the form

$$
h(x, y)=(P(x)-a y, x)
$$

where $p$ is a polynomial of $x$ of degree at least 2 and $a \neq 0$.

It seems to be difficult to extend Jung's Theorem to $\mathbb{C}^{n}$ for $n \geq 3$. So we cannot follow Friedland and Milnor's proof to classify polynomial automorphisms in higher dimensions. But if we restrict to polynomials of degree at most 2 in $\mathbb{C}^{2}$, it is not necessary to apply Jung's Theorem for the classification, see $[\mathbf{H O}]$ for a proof. In this paper, we give the classification of degree at most 2 polynomial self-maps of $\mathbb{C}^{3}$ with nowhere vanishing Jacobian determinant up to affine conjugation. The organization of this paper is as follows: in Section 2, we give the statement of our classification Theorem 2.1 and we also include some remarks on the dynamical differences between the various classes in our theorem. In Section 3, the proof of Theorem 2.1 is given and in Section 4, we briefly give some discussions of some basic dynamical properties of these maps.

\section{The statement of main theorem and some remarks}

Theorem 2.1. If $f: \mathbb{C}^{3} \rightarrow \mathbb{C}^{3}$ is a degree at most 2 polynomial selfmap with nowhere vanishing Jacobian determinant, then $f$ is affinely conjugate to one of the following maps:

(1) An affine automorphism;

(2) An elementary polynomial automorphism

$$
E(x, y, z)=(P(y, z)+a x, Q(z)+b y, c z+d),
$$


where $P$ is a polynomial of $y, z$ of degree at most $2, Q$ is a polynomial of $z$ of degree at most 2 and $a b c \neq 0$. Note that it maps every hyperplane $z=k$ to a hyperplane $z=k^{\prime}$ and maps every line $y=k_{1}, z=k_{2}$ to a line $y=k_{1}^{\prime}, z=k_{2}^{\prime}$;

(3)

$$
\left\{\begin{array}{l}
H_{1}(x, y, z)=(P(x, z)+a y, Q(z)+x, c z+d) \\
H_{2}(x, y, z)=(P(y, z)+a x, Q(y)+b z, y) \\
H_{3}(x, y, z)=(P(x, z)+a y, Q(x)+z, x) \\
H_{4}(x, y, z)=(P(x, y)+a z, Q(y)+x, y) \\
H_{5}(x, y, z)=(P(x, y)+a z, Q(x)+b y, x)
\end{array}\right.
$$

where $P$ and $Q$ are polynomials with $\max \{\operatorname{deg}(P), \operatorname{deg}(Q)\}=2$ and $a b c \neq 0$.

\section{Remark 2.2.}

$$
\left\{\begin{aligned}
H_{1}^{-1}(x, y, z)= & \left(y-Q\left(\frac{1}{c}(z-d)\right),\right. \\
& \left.\frac{1}{a}\left[x-P\left(y-Q\left(\frac{1}{c}(z-d)\right), \frac{1}{c}(z-d)\right)\right], \frac{1}{c}(z-d)\right) \\
H_{2}^{-1}(x, y, z)= & \left(\frac{1}{a}\left[x-P\left(z, \frac{1}{b}(y-Q(z))\right)\right], z, \frac{1}{b}[y-Q(z)]\right) \\
H_{3}^{-1}(x, y, z)= & \left(z, \frac{1}{a}[x-P(z, y-Q(z))], y-Q(z)\right) \\
H_{4}^{-1}(x, y, z)= & \left(y-Q(z), z, \frac{1}{a}[x-P(y-Q(z), z)], z\right) \\
H_{5}^{-1}(x, y, z)= & \left(z, \frac{1}{b}[y-Q(z)], \frac{1}{a}\left[x-P\left(z, \frac{1}{b}(y-Q(z))\right)\right]\right) .
\end{aligned}\right.
$$

Remark 2.3. If $\tau(x, y, z)=(y, z, x)$, then $H_{4}=H_{3} \circ \tau, H_{2}=H_{5} \circ \tau$, $H_{1}=H_{5} \circ \tau^{2}$.

Remark 2.4. Some Generic Dynamical differences between the various classes: Assume for simplicity that the constants $|a|,|b|,|c|<1$.

First of all the elementary maps and the class $H_{1}$ distinguish themselves from the other classes by the fact that the maps fix a hypersurface and the orbits of all points outside this hypersurface converge to it. Hence the dynamics reduces to two dimensions. In the case of $H_{1}$, the 
maps reduces to a Hénon map on the fixed hypersurface $z=\alpha$. In the case of the elementary maps, the orbits in the fixed hypersurface $z=\alpha$ converge to the fixed curve $y=\beta$ on which the maps are automorphisms. In fact both the elementary maps and the maps of class $H_{1}$ are semidirect products over a mapping $A(z)=c z+d$, i.e. there is a function $z \rightarrow f_{z}(x, y) \in \operatorname{Aut}\left(\mathbb{C}^{2}\right)$ such that $F(x, y, z)=\left(f_{z}(x, y), A(z)\right)$.

Hence we need only to find dynamical differences between the classes $\mathrm{H}_{2}, \mathrm{H}_{3}, \mathrm{H}_{4}, \mathrm{H}_{5}$.

First we can observe that it is natural to consider the maps $H_{2}, H_{3}$ together as opposed to the maps $H_{4}, H_{5}$. There is a dynamical difference in the asymptotic dynamics. For the maps $\mathrm{H}_{2}, \mathrm{H}_{3}$, the orbits generically converge to one point at infinity. For example, for the map $H_{3}$, if $P(x, y)=A x^{2}+\cdots, Q(x)=B x^{2}+\cdots$, then this is the point $[A: B: 0: 0]$ at infinity in projective coordinates. On the other hand, for the maps $H_{4}, H_{5}$ the generic orbit converges to a complex line at infinity.

It remains to distinguish dynamically the maps $H_{2}$ and $H_{3}$ as well as to distinguish the maps $H_{4}$ and $H_{5}$.

Comparing the maps $\mathrm{H}_{2}$ and $\mathrm{H}_{3}$, we observe that the map $\mathrm{H}_{2}$ is a Hénon map in the last two coordinates, $(y, x) \rightarrow(Q(y)+b z, y)$. In other words such a map $F$ is a semi-direct product over a mapping $h(y, z) \in$ $\operatorname{Aut}\left(\mathbb{C}^{2}\right)$, i.e. there is an analytic function $(y, z) \rightarrow A_{y, z} \in \operatorname{Aut}(\mathbb{C})$ such that $F(x, y, z)=\left(A_{y, z}(x), h(y, z)\right)$. This sets $H_{2}$ apart from $H_{3}$.

Comparing the maps $H_{4}$ and $H_{5}$ we consider again their behaviour at infinity. We see that there is a $P^{1}$ at infinity which is mapped to itself. For $H_{4}$ this map is a second degree polynomial, while for $H_{5}$ this map is rational of degree 2, i.e. has a more complicated dynamics.

\section{The Proof of Theorem 2.1}

Let $G$ be the family of degree at most 2 polynomial self-map of $\mathbb{C}^{3}$ with nowhere vanishing Jacobian determinant. For any $f \in G$, we can write $f$ in the following form:

$$
f(x, y, z)=\left(f_{1}(x, y, z), f_{2}(x, y, z), f_{3}(x, y, z)\right) .
$$

Because the degree of $f$ is at most 2, the Jacobian matrix of $f$ is as follows:

$$
f^{\prime}(x, y, z)=\left(\begin{array}{lll}
w_{1}(x, y, z) & w_{2}(x, y, z) & w_{3}(x, y, z) \\
w_{4}(x, y, z) & w_{5}(x, y, z) & w_{6}(x, y, z) \\
w_{7}(x, y, z) & w_{8}(x, y, z) & w_{9}(x, y, z)
\end{array}\right)
$$

where $w_{j}(x, y, z)=a_{j} x+b_{j} y+c_{j} z+d_{j}$ for $1 \leq j \leq 9$. 
Since the determinant of the Jacobian matrix $f^{\prime}(x, y, z)$ is a nonzero constant, all coefficients of the polynomial $\operatorname{det}\left(f^{\prime}\right)$ must be zero except the constant. In particular, the coefficients of $x^{3}, y^{3}$ and $z^{3}$ must be zero, i.e.,

$$
\operatorname{det}(A)=\operatorname{det}(B)=\operatorname{det}(C)=0
$$

where

(2) $A=\left(\begin{array}{lll}a_{1} & a_{2} & a_{3} \\ a_{4} & a_{5} & a_{6} \\ a_{7} & a_{8} & a_{9}\end{array}\right), B=\left(\begin{array}{lll}b_{1} & b_{2} & b_{3} \\ b_{4} & b_{5} & b_{6} \\ b_{7} & b_{8} & b_{9}\end{array}\right), C=\left(\begin{array}{lll}c_{1} & c_{2} & c_{3} \\ c_{4} & c_{5} & c_{6} \\ c_{7} & c_{8} & c_{9}\end{array}\right)$.

It is easy to see that both $b_{1}$ and $a_{2}$ are the coefficient of $x y$ in $f_{1}(x, y, z)$, this implies that $b_{1}=a_{2}$. By looking at the coefficients of $x y$, $y z$, and $x z$ in $f=\left(f_{1}, f_{2}, f_{3}\right)$, we obtain the following table:

\begin{tabular}{|l|l|l|}
\hline$b_{1}=a_{2}$ & $c_{1}=a_{3}$ & $c_{2}=b_{3}$ \\
\hline$b_{4}=a_{5}$ & $c_{4}=a_{6}$ & $c_{5}=b_{6}$ \\
\hline$b_{7}=a_{8}$ & $c_{7}=a_{9}$ & $c_{8}=b_{9}$ \\
\hline
\end{tabular}

Table (ABC)

By using the above table we can write our function $f$ in the following form:

(3) $f(x, y, z)=\left(\phi_{1}(x, y, z)+L_{1}(x, y, z), \phi_{2}(x, y, z)\right.$

$$
\left.+L_{2}(x, y, z), \phi_{3}(x, y, z)+L_{3}(x, y, z)\right)
$$

where

$$
\left\{\begin{array}{l}
\phi_{1}(x, y, z)=\frac{1}{2} a_{1} x^{2}+\frac{1}{2} b_{2} y^{2}+\frac{1}{2} c_{3} z^{2}+b_{1} x y+c_{2} y z+c_{1} x z \\
\phi_{2}(x, y, z)=\frac{1}{2} a_{4} x^{2}+\frac{1}{2} b_{5} y^{2}+\frac{1}{2} c_{6} z^{2}+b_{4} x y+c_{5} y z+c_{4} x z \\
\phi_{3}(x, y, z)=\frac{1}{2} a_{7} x^{2}+\frac{1}{2} b_{8} y^{2}+\frac{1}{2} c_{9} z^{2}+b_{7} x y+c_{8} y z+c_{7} x z \\
L_{1}(x, y, z)=d_{1} x+d_{2} y+d_{3} z+e_{1} \\
L_{2}(x, y, z)=d_{4} x+d_{5} y+d_{6} z+e_{2} \\
L_{3}(x, y, z)=d_{7} x+d_{8} y+d_{9} z+e_{3} .
\end{array}\right.
$$


Let's introduce the following trivial lemma which is useful in our proof of Theorem 2.1.

Lemma 3.1. Let $F=\left(f_{1}, \ldots, f_{n}\right)$ be a polynomial self-map of $\mathbb{C}^{n}$ of degree at most 2 , with nowhere vanishing Jacobian. Let $g$ and $h$ be any affine automorphisms of $\mathbb{C}^{n}$. We denote the degree 2 homogeneous part of $F$ by $\left(\phi_{1}, \ldots, \phi_{n}\right)$ and the degree 2 homogeneous part of $g \circ F \circ h$ by $\left(\psi_{1}, \ldots, \psi_{n}\right)$. Then the following statements are equivalent:

(i) There exist constants $\alpha_{1}, \ldots, \alpha_{n}$ with $\sum_{j=1}^{n}\left|\alpha_{j}\right| \neq 0$ such that

$$
\sum_{j=1}^{n} \alpha_{j} \phi_{j} \equiv 0,
$$

(ii) There exist constants $\beta_{j}$ with $\sum_{j=1}^{n}\left|\beta_{j}\right| \neq 0$ such that

$$
\sum_{j=1}^{n} \beta_{j} \psi_{j}=0 .
$$

\section{Proof: Clear.}

Remark 3.2. For the map $F$ in above lemma, if we want to prove that $\sum_{j=1}^{n} \alpha_{j} \phi_{j} \equiv 0$, we can simplify $J_{F}$, the Jacobian matrix of $F$, by composing constant invertible matrices in both sides of $J_{F}$. Note that $J_{(g \circ F \circ h)}=J_{g}(F(h)) J_{F}(h) J_{h}=J_{g} J_{F}(h) J_{h}$, i.e., we have to use the new variables for the Jacobian matrix of $F$, but this doesn't matter because $J_{h}$ is a constant matrix and therefore we may keep the original notation as the new variables.

Let's recall the following result from $[\mathbf{H O}]$ :

Lemma 3.3. Let $f(x, y)=\left(f_{1}(x, y), f_{2}(x, y)\right)=\left(P_{1}(x, y)+A_{1}(x, y)\right.$, $\left.P_{2}(x, y)+A_{2}(x, y)\right)$ be a polynomial self-map of $\mathbb{C}^{2}$ of degree at most 2 , with nowhere vanishing Jacobian, where $P_{j}(x, y)$ is the corresponding degree 2 homogeneous polynomial of $f_{j}$ and $A_{j}=f_{j}-P_{j}$. Then the homogeneous polynomials $P_{1}$ and $P_{2}$ are proportional.

Lemma 3.4. If for all constants $\alpha, \beta$ and $\gamma$ with $|\alpha|+|\beta|+|\gamma| \neq 0$, we have $\alpha \phi_{1}+\beta \phi_{2}+\gamma \phi_{3} \not \equiv 0$, then there exist affine automorphisms $g$ and $h$ such that

$$
\psi_{1}=x^{2}, \quad \psi_{2}=x y, \quad \psi_{3}=y^{2},
$$

where $\left(\psi_{1}, \psi_{2}, \psi_{3}\right)$ is the degree 2 homogeneous part of the map $g \circ f \circ h$.

Proof: Let $\phi=\left[\phi_{1}: \phi_{2}: \phi_{3}\right]: P^{2} \rightarrow P^{2}$. Since the Jacobian determinant of $\phi$ is 0 , the rank of $\phi$ is at most 1 . If $\phi_{1} \equiv 0$, then 
$1 \phi_{1}+0 \phi_{2}+0 \phi_{3} \equiv 0$. So WLOG, we may assume that $\phi_{j} \not \equiv 0$ for $j=1,2,3$.

(i) If the rank of $\phi$ is 0 , i.e., $\phi$ is constant, then we may assume that $\left[\phi_{1}: \phi_{2}: \phi_{3}\right]=[1: 0: 0]$. In this case, we have

$$
0 \phi_{1}+1 \phi_{2}+0 \phi_{3} \equiv 0
$$

(ii) If rank of $\phi$ is 1 , we may assume that $\phi([1: 0: 0])=[1: 0: 0]$ and that $\phi([x: 0: 1])$ is non-constant.

Let

$\left.\left(\phi_{1}, \phi_{2}, \phi_{3}\right)\right|_{[x: 0: 1]}=\left(a_{1} x^{2}+b_{1} x+c_{1}, a_{2} x^{2}+b_{2} x+c_{2}, a_{3} x^{3}+b_{3} x+c_{3}\right)$.

Then since $\phi([1: 0: 0])=[1: 0: 0], a_{1} \neq 0, a_{2}=a_{3}=0$.

Hence

$$
\left.\left(\phi_{1}, \phi_{2}, \phi_{3}\right)\right|_{[x: 0: 1]}=\left(a_{1} x^{2}+b_{1} x+c_{1}, b_{2} x+c_{2}, b_{3} x+c_{3}\right) .
$$

Since $\phi([x: 0: 1])$ is non-constant, it follows that $b_{2}$ or $b_{3} \neq 0$. By Lemma 3.1 we may assume that $b_{2} \neq 0$ and $b_{1}=b_{3}=0$.

Hence

$$
\left.\left(\phi_{1}, \phi_{2}, \phi_{3}\right)\right|_{x: 0: 1]}=\left(a_{1} x^{2}+c_{1}, b_{2} x+c_{2}, c_{3}\right) .
$$

If $c_{3}=0$, then we have $\phi_{3}=0$, so we have $0 \phi_{1}+0 \phi_{2}+1 \phi_{3} \neq 0$, which is impossible. Hence $c_{3} \neq 0$, so we may assume that

$$
\left.\left(\phi_{1}, \phi_{2}, \phi_{3}\right)\right|_{[x: 0: 1]}=\left(x^{2}, x, 1\right) .
$$

Hence $\phi(y=0)=\left(X Z=Y^{2}\right)$. Sice $\phi$ has rank $1, \phi\left(P^{2}\right) \subset(X Z=$ $\left.Y^{2}\right)$, so

$$
\phi_{1} \phi_{3} \equiv \phi_{2}^{2}
$$

(1) If $\phi_{1}=c \phi_{2}$ for a nonzero constant $c$, then $\phi_{1}-c^{-1} \phi_{2}+0 \phi_{3} \equiv 0$.

(2) If $\phi_{3}=c \phi_{2}$ for a nonzero constant $c$, then we have $0 \phi_{1}+c^{-1} \phi_{2}-$ $\phi_{3} \equiv 0$.

(3) Hence $\phi_{1} \neq c_{1} \phi_{2}$ and $\phi_{3} \neq c_{2} \phi_{2}$ for any constants $c_{1}$ and $c_{2}$. Then $\phi_{2}$ must be a product of two nonproportional linear factors, $\phi=L_{1} L_{2}$. This implies that $\phi_{1}=c_{1} L_{1}^{2}$ and $\phi_{3}=c_{3} L_{2}^{2}$ or vice versa. Setting $L_{1}=x$ and $L_{2}=y$ and scaling we finish the proof of the lemma. 
Lemma 3.5. There exist constants $\alpha, \beta$ and $\gamma$ such that $|\alpha|+|\beta|+$ $|\gamma| \neq 0$ and

$$
\alpha \phi_{1}+\beta \phi_{2}+\gamma \phi_{3} \equiv 0
$$

Proof: If for all constants $\alpha, \beta$ and $\gamma$ with $|\alpha|+|\beta|+|\gamma| \neq 0, \alpha \phi_{1}+$ $\beta \phi_{2}+\gamma \phi_{3} \not \equiv 0$, then by Lemma 3.1 and Lemma 3.4 we may assume that $\psi_{1}=x^{2}, \psi_{2}=x y, \psi_{3}=y^{2}$.

In this case, the Jacobian matrix of $f$ is as follows:

$$
f^{\prime}(x, y, z)=\left(\begin{array}{ccc}
2 x+d_{1} & d_{2} & d_{3} \\
y+d_{4} & x+d_{5} & d_{6} \\
d_{7} & 2 y+d_{8} & d_{9}
\end{array}\right)
$$

Since the Jacobian determinant is a nonzero constant, then

$$
\operatorname{det}\left(\begin{array}{ccc}
2 x+d_{1} & d_{2} & d_{3} \\
y+d_{4} & x+d_{5} & d_{6} \\
d_{7} & 2 y+d_{8} & d_{9}
\end{array}\right)=\operatorname{det}\left(\begin{array}{ccc}
d_{1} & d_{2} & d_{3} \\
d_{4} & d_{5} & d_{6} \\
d_{7} & d_{8} & d_{9}
\end{array}\right)=\text { const. } \neq 0
$$

But

$$
\operatorname{det}\left(\begin{array}{ccc}
2 x+d_{1} & d_{2} & d_{3} \\
y+d_{4} & x+d_{5} & d_{6} \\
d_{7} & 2 y+d_{8} & d_{9}
\end{array}\right)=2 d_{9} x^{2}-4 d_{6} x y+2 d_{3} y^{2}+\cdots
$$

This implies that $d_{3}=d_{6}=d_{9}=0$, i.e.,

$$
\operatorname{det}\left(\begin{array}{lll}
d_{1} & d_{2} & d_{3} \\
d_{4} & d_{5} & d_{6} \\
d_{7} & d_{8} & d_{9}
\end{array}\right)=0
$$

This is a contradiction.

Proof of the Theorem 2.1: If $\operatorname{deg}(f)=1$, then it is easy to see that $f$ is an affine automorphism.

If $\operatorname{deg}(f)=2$, then by Lemma 3.5 we can assume that there exist constants $k_{1}$ and $k_{2}$ such that

$$
\phi_{3}=k_{1} \phi_{1}+k_{2} \phi_{2}
$$

Then

$$
f=\left(\phi_{1}+L_{1}, \phi_{2}+L_{2}, k_{1} \phi_{1}+k_{2} \phi_{2}+L_{3}\right) .
$$


Let $g_{1}(x, y, z)=\left(x, y, z-k_{1} x-k_{2} y\right)$, then $g_{1}^{-1}(x, y, z)=(x, y, z+$ $\left.k_{1} x+k_{2} y\right)$. Then

$$
\begin{aligned}
F_{1}(x, y, z):= & g_{1} \circ f \circ g_{1}^{-1}(x, y, z) \\
= & g_{1} \circ f\left(x, y, z+k_{1} x+k_{2} y\right) \\
= & \left(\phi_{4}(x, y, z)+L_{4}(x, y, z), \phi_{5}(x, y, z)\right. \\
& \left.+L_{5}(x, y, z), L_{6}(x, y, z)\right)
\end{aligned}
$$

where $\phi_{j}$ are degree 2 homogeneous polynomial and $L_{j}(x, y, z)$ are degree 1 polynomial and written as $L_{j}(x, y, z):=\alpha_{j} x+\beta_{j} y+\gamma_{j} z+\rho_{j}$. In particular, $\left|\alpha_{6}\right|+\left|\beta_{6}\right|+\left|\gamma_{6}\right| \neq 0$.

We will classify $L_{6}$ into the following 3 cases:

Case (i): $\alpha_{6} \neq 0$.

Let $g_{2}(x, y, z)=\left(\alpha_{6} x+\beta_{6} y+\gamma_{6} z+\rho_{6}, y, z\right)$, then $g_{2}^{-1}(x, y, z)=$ $\left(\frac{1}{\alpha_{6}}\left(x-\beta_{6} y-\gamma_{6} z-\rho_{6}\right), y, z\right)$. Then

$$
\begin{aligned}
F_{2}(x, y, z): & =g_{2} \circ F_{1} \circ g_{2}^{-1}(x, y, z) \\
& =\left(\phi_{7}(x, y, z)+L_{7}(x, y, z), \phi_{8}(x, y, z)+L_{8}(x, y, z), x\right)
\end{aligned}
$$

where $\phi_{j}$ are degree 2 homogeneous polynomials and $L_{j}(x, y, z)$ are degree 1 polynomials and written as $L_{j}(x, y, z):=\alpha_{j} x+\beta_{j} y+\gamma_{j} z+\rho_{j}$.

Then the Jacobian matrix of $F_{2}$ has the following form:

$$
F_{2}^{\prime}(x, y, z)=\left(\begin{array}{ccc}
\left(\phi_{7}+L_{7}\right)_{x}^{\prime} & \left(\phi_{7}+L_{7}\right)_{y}^{\prime} & \left(\phi_{7}+L_{7}\right)_{z}^{\prime} \\
\left(\phi_{8}+L_{8}\right)_{x}^{\prime} & \left(\phi_{8}+L_{8}\right)_{y}^{\prime} & \left(\phi_{8}+L_{8}\right)_{z}^{\prime} \\
1 & 0 & 0
\end{array}\right)
$$

Then since $\operatorname{det}\left(F_{2}^{\prime}(x, y, z)\right)$ is a nonzero constant,

$$
\operatorname{det}\left(\begin{array}{cc}
\left(\phi_{7}+L_{7}\right)_{y}^{\prime} & \left(\phi_{7}+L_{7}\right)_{z}^{\prime} \\
\left(\phi_{8}+L_{8}\right)_{y}^{\prime} & \left(\phi_{8}+L_{8}\right)_{z}^{\prime}
\end{array}\right)=a \text { nonzero constant. }
$$

Then for any fixed $x$, we may consider

$$
F_{x}(y, z)=\left(\phi_{7}+L_{7}, \phi_{8}+L_{8}\right)
$$

as a degree 2 polynomial self-map of $\mathbb{C}^{2}$ with nowhere vanishing Jacobian determinant.

We can write

$$
\begin{aligned}
& \phi_{7}+L_{7}=a_{7} x^{2}+b_{7} y^{2}+c_{7} z^{2}+d_{7} y z+e_{7} x y+f_{7} x z+\cdots \\
& \phi_{8}+L_{8}=a_{8} x^{2}+b_{8} y^{2}+c_{8} z^{2}+d_{8} y z+e_{8} x y+f_{8} x z+\cdots
\end{aligned}
$$


By Lemma 3.3 it follows that the homogeneous polynomials $b_{7} y^{2}+$ $c_{7} z^{2}+d_{7} y z$ and $b_{8} y^{2}+c_{8} z^{2}+d_{8} y z$ are proportional. Moreover we make the following claim:

Claim. The homogeneous polynomials $b_{7} y^{2}+c_{7} z^{2}+d_{7} y z+e_{7} x y+f_{7} x z$ and $b_{8} y^{2}+c_{8} z^{2}+d_{8} y z+e_{8} x y+f_{8} x z$ are also proportional.

$$
J_{F_{x}}(y, z)=\left(\begin{array}{ll}
2 b_{7} y+d_{7} z+e_{7} x+k_{1} & 2 c_{7} z+d_{7} y+f_{7} x+k_{2} \\
2 b_{8} y+d_{8} z+e_{8} x+k_{3} & 2 c_{8} z+d_{8} y+f_{8} x+k_{4}
\end{array}\right)
$$

and $\operatorname{det} J_{F_{x}}(y, z)=a$ nonzero constant independent on $x$.

(1) Since $b_{7} y^{2}+c_{7} z^{2}+d_{7} y z$ and $b_{8} y^{2}+c_{8} z^{2}+d_{8} y z$ are proportional, we may compose an invertible constant matrix, say $M_{1}$, to the left of the matrix of $J_{F_{x}}(y, z)$ to kill the $y, z$ terms in the first row or second row. WLOG, we may assume that we killed the $y, z$ terms in the first row. Now our Jacobian matrix becomes

$$
M_{1} J_{F_{x}}(y, z)=\left(\begin{array}{cc}
e_{7} x+k_{1} & f_{7} x+k_{2} \\
2 b_{8} y+d_{8} z+e_{8} x+k_{3} & 2 c_{8} z+d_{8} y+f_{8} x+k_{4}
\end{array}\right) .
$$

Note that $e_{7}, k_{1}, f_{7}, k_{2}$ are different from the original values, for simplicity we still use the same notation. The following discussion will follow the same rule.

(2) If $e_{7}=f_{7}=0$, then we are done.

(3) If $e_{7} \neq 0, f_{7} \neq 0$, then we may compose an invertible constant matrix to the right of the matrix $M_{1} J_{F_{x}}$ to kill $f_{7}$. Now the matrix becomes

$$
\left(\begin{array}{cc}
e_{7} x+k_{1} & k_{2} \\
2 b_{8} y+d_{8} z+e_{8} x+k_{3} & 2 c_{8} z+d_{8} y+f_{8} x+k_{4}
\end{array}\right) .
$$

Since the determinant of this matrix is a nonzero constant, we have $c_{8}=d_{8}=f_{8}=0$. If $e_{8} \neq 0$, we will use the fact $e_{7} \neq 0$ to kill $e_{8}$. So now the matrix ix

$$
\left(\begin{array}{cc}
e_{7} x+k_{1} & k_{2} \\
2 b_{8} y+k_{3} & k_{4}
\end{array}\right)
$$

The determinant of this matrix is $e_{7} k_{4} x-2 b_{8} y k_{2}+k_{1} k_{4}-k_{2} k_{3}$ which is a nonzero constant. This implies that $e_{7} k_{4}=0, b_{8} k_{2}=0 . \quad e_{7} \neq 0$ implies that $k_{4}=0 . k_{2}$ cannot be 0 otherwise the determinant is 0 , so $b_{8}=0$. 
Now our matrix is pretty simple:

$$
\left(\begin{array}{cc}
e_{7} x+k_{1} & k_{2} \\
k_{3} & 0
\end{array}\right) .
$$

It is easy to see that our claim is true for the map $F_{x}$ with above matrix as its Jacobian matrix. By Lemma 3.1 and Remark 3.2, we proved our claim.

(4) If one of $\left\{e_{7}, f_{7}\right\}$ is 0 and the other is different from 0 , then go to the case $(3)$.

By the above claim we can write

$$
\begin{aligned}
& \phi_{7}+L_{7}=a_{7} x^{2}+\psi_{7}(x, y, z)+L_{7}(x, y, z) \\
& \phi_{8}+L_{8}=a_{8} x^{2}+\psi_{8}(x, y, z)+L_{8}(x, y, z)
\end{aligned}
$$

where $\psi_{7}(x, y, z)=b_{7} y^{2}+c_{7} z^{2}+d_{7} y z+e_{7} x y+f_{7} x z$ and $\psi_{8}(x, y, z)=$ $b_{8} y^{2}+c_{8} z^{2}+d_{8} y z+e_{8} x y+f_{8} x z$ are proportional. This implies that either (i-a) $\psi_{8} \equiv k \psi_{7}$ for some constant $k$; or (i-b) $\psi_{7} \equiv 0$.

Case $(i-a): \psi_{8}=k \psi_{7}$ for some constant $k$.

Let $g_{3}(x, y, z)=(x, y-k x, z)$, then $g_{3}^{-1}(x, y, z)=(x, y+k x, z)$,

$$
\begin{aligned}
F_{3}(x, y, z):= & g_{3} \circ F_{2} \circ g_{3}^{-1}(x, y, z) \\
= & \left(a_{7} x^{2}+\psi_{7}(x, y+k x, z)+L_{7}(x, y+k x, z), a_{8}^{\prime} x^{2}\right. \\
& \left.+L_{8}^{\prime}(x, y, z), x\right) \\
= & \left(p_{1}(x)+\psi(x, y, z)+l_{7}(y, z), p_{2}(x)+l_{8}(y, z), x\right)
\end{aligned}
$$

where $a_{8}^{\prime}$ is the new coefficient of $x^{2}$ after the compositions, $L_{8}^{\prime}$ is the new linear function, $p_{1}, p_{2}$ are the polynomial of $x$ of the corresponding coordinates, $l_{7}, l_{8}$ are linear functions of $y, z$ of the corresponding coordinates, $\psi(x, y, z)$ is the degree 2 homogeneous polynomial except the $x^{2}$ term in $a_{7} x^{2}+\psi_{7}(x, y+k x, z)+L_{7}(x, y+k x, z)$.

Now we assume that the linear function $l_{8}(y, z)=\alpha_{8} y+\beta_{8} z$ with $\left|\alpha_{8}\right|+\left|\beta_{8}\right| \neq 0$.

If $\alpha_{8} \neq 0$, let $g_{4}(x, y, z)=\left(x, \alpha_{8} y+\beta_{8} z, z\right)$, then

$$
\begin{aligned}
H_{3}(x, y, z):= & g_{4} \circ F_{3} \circ g_{4}^{-1}(x, y, z) \\
= & \left(p_{1}(x)+\psi\left(x, \frac{1}{\alpha_{8}}\left(y-\beta_{8} z\right), z\right)\right. \\
& \left.+l_{7}\left(\frac{1}{\alpha_{8}}\left(y-\beta_{8} z\right), z\right), \alpha_{8}\left(p_{2}(x)+y\right)+\beta_{8} x, x\right) .
\end{aligned}
$$


Since the Jacobian determinant is a nonzero constant, $\frac{\partial}{\partial z}\left(p_{1}(x)+\right.$ $\left.\psi\left(x, \frac{1}{\alpha_{8}}\left(y-\beta_{8} z\right), z\right)+l_{7}\left(\frac{1}{\alpha_{8}}\left(y-\beta_{8} z\right), z\right)\right)=$ nonzero constant. So we may rewrite $H_{3}$ in the following form:

$$
H_{3}(x, y, z)=(P(x, y)+a z, Q(x)+b y, x)
$$

where $P$ and $Q$ are polynomials with $\max \{\operatorname{deg}(P), \operatorname{deg}(Q)\}=2$ and $a \neq 0$.

If $\alpha_{8}=0$, then $\beta_{8} \neq 0$. In this case, following a similar discussion as above, we may rewrite our map $F_{3}$ as the following form:

$$
F_{3}(x, y, z)=\left(p(x, z)+c y, q(x)+\beta_{8} z, x\right)
$$

where $p(x, z)$ is a degree at most 2 polynomial of $x$ and $z$.

Let $g(x, y, z)=\left(\beta_{8} x, y, \beta_{8} z\right)$ and let $H_{1}=g \circ F_{3} \circ g^{-1}$, then $H_{1}$ has the following form:

$$
H_{3}(x, y, z)=(P(x, z)+a y, Q(x)+z, x)
$$

where $P$ and $Q$ are polynomials with $\max \{\operatorname{deg}(P), \operatorname{deg}(Q)\}=2$ and $a \neq 0$.

Case $(i-b): \psi_{7} \equiv 0$.

In this case, $F_{2}(x, y, z)=\left(p_{1}(x)+l_{7}(y, z), a_{8} x^{2}+\psi_{8}+L_{8}, x\right)$ where $p_{1}(x)$ is polynomial of $x$ and $l_{7}(x, y)=\alpha_{7} y+\beta_{7} z$.

If $\alpha_{7} \neq 0$, let $g(x, y, z)=\left(x, \alpha_{7} y+\beta_{7} z, z\right)$, since the Jacobian determinant of $g \circ F_{2} \circ g^{-1}$ is a nonzero constant, it is easy to check that $g \circ F_{2} \circ g^{-1}$ has the following form:

$$
G_{2}(x, y, z)=(q(x)+y, p(x, y)+a z, x)
$$

where $P$ and $Q$ are polynomials with $\max \{\operatorname{deg}(P), \operatorname{deg}(Q)\}=2$ and $a \neq 0$.

If $\alpha_{7}=0$, then $\beta_{7} \neq 0$. In this case, $F_{2}=\left(p_{1}(x)+\beta_{7} z, a_{8} x^{2}+\psi_{8}+\right.$ $\left.L_{8}, x\right)$. Using the fact that the Jacobian determinant of $F_{2}$ is a nonzero constant, we have that $\frac{\partial}{\partial y}\left(a_{8} x^{2}+\psi_{8}+L_{8}\right)=$ nonzero constant. So we can rewrite $F_{2}$ in the following form:

$$
G_{4}(x, y, z)=(q(x)+b z, p(x, z)+a y, x)
$$

where $P$ and $Q$ are polynomials with $\max \{\operatorname{deg}(P), \operatorname{deg}(Q)\}=2$ and $a b \neq 0$. 
Let $g(x, y, z)=(y, x, z)$, then

$$
\begin{aligned}
& H_{4}:=g \circ G_{2} \circ g^{-1}=(P(x, y)+a z, Q(y)+x, y) \\
& H_{2}:=g \circ G_{4} \circ g^{-1}=(P(y, z)+a x, Q(y)+b z, y)
\end{aligned}
$$

where $P$ and $Q$ are polynomials with $\max \{\operatorname{deg}(P), \operatorname{deg}(Q)\}=2$ and $a b \neq 0$.

Therefore, in the case (i) the map $F_{2}$ is affinely conjugate to one of the maps $\mathrm{H}_{2}, \mathrm{H}_{3}, \mathrm{H}_{4}, \mathrm{H}_{5}$.

Case (ii): $\alpha_{6}=0$ and $\beta_{6} \neq 0$.

In this case, we construct the following affine map $g_{5}$ :

$$
g_{5}(x, y, z)=\left(x, \beta_{6} y+\gamma_{6} z+\rho_{6}, z\right) .
$$

Then $g_{5}^{-1}(x, y, z)=\left(x, \frac{1}{\beta_{6}}\left(y-\gamma_{6} z-\rho_{6}\right), z\right)$.

$$
\begin{aligned}
F_{5}(x, y, z): & =g_{5} \circ F_{1} \circ g_{5}^{-1}(x, y, z) \\
& =\left(\phi_{9}+L_{9}, \phi_{10}+L_{10}, y\right)
\end{aligned}
$$

where $\phi_{j}$ are degree 2 homogeneous polynomials and $L_{j}(x, y, z)$ are degree 1 polynomials and written as $L_{j}(x, y, z):=\alpha_{j} x+\beta_{j} y+\gamma_{j} z+\rho_{j}$.

Then the Jacobian matrix of $F_{5}$ has the following form:

$$
F_{5}^{\prime}(x, y, z)=\left(\begin{array}{ccc}
\left(\phi_{9}+L_{9}\right)_{x}^{\prime} & \left(\phi_{9}+L_{9}\right)_{y}^{\prime} & \left(\phi_{9}+L_{9}\right)_{z}^{\prime} \\
\left(\phi_{10}+L_{10}\right)_{x}^{\prime} & \left(\phi_{10}+L_{10}\right)_{y}^{\prime} & \left(\phi_{10}+L_{10}\right)_{z}^{\prime} \\
0 & 1 & 0
\end{array}\right)
$$

The property that $\operatorname{det}\left(F_{5}^{\prime}(x, y, z)\right)=a$ nonzero constant implies that

$$
\operatorname{det}\left(\begin{array}{cc}
\left(\phi_{9}+L_{9}\right)_{x}^{\prime} & \left(\phi_{9}+L_{9}\right)_{z}^{\prime} \\
\left(\phi_{10}+L_{10}\right)_{x}^{\prime} & \left(\phi_{10}+L_{10}\right)_{z}^{\prime}
\end{array}\right)=a \text { nonzero constant. }
$$

Then following the same arguments in the proof of case (i), we can prove that $F_{5}$ is conjugate to one of the following maps:

$$
\begin{aligned}
& H_{2}(x, y, z)=(P(y, z)+a x, Q(y)+b z, y) \\
& H_{4}(x, y, z)=(P(x, y)+a z, Q(y)+x, y) \\
& G_{3}(x, y, z)=(q(y)+z, p(y, z)+a x, y) \\
& G_{5}(x, y, z)=(q(y)+b x, p(x, y)+a z, y)
\end{aligned}
$$


where $P$ and $Q$ are polynomials with $\max \{\operatorname{deg}(P), \operatorname{deg}(Q)\}=2$ and $a b \neq 0$.

But $G_{j}$ is conjugate to $H_{j}$ by the affine map $g(x, y, z)=(y, x, z)$ for $j=3,5$. So $F_{5}$ is affinely conjugate to one of the maps $H_{2}, H_{3}, H_{4}, H_{5}$.

Case (iii): If $\alpha_{6}=\beta_{6}=0$.

In this case, $\gamma_{6} \neq 0$. Then we have

$$
F_{1}(x, y, z)=\left(\phi_{4}+L_{4}, \phi_{5}+L_{5}, \gamma_{6} z+\rho_{6}\right) .
$$

Then the Jacobian matrix of $F_{1}$ has the following form:

$$
F_{1}^{\prime}(x, y, z)=\left(\begin{array}{ccc}
\left(\phi_{4}+L_{4}\right)_{x}^{\prime} & \left(\phi_{4}+L_{4}\right)_{y}^{\prime} & \left(\phi_{4}+L_{4}\right)_{z}^{\prime} \\
\left(\phi_{5}+L_{5}\right)_{x}^{\prime} & \left(\phi_{5}+L_{5}\right)_{y}^{\prime} & \left(\phi_{5}+L_{5}\right)_{z}^{\prime} \\
0 & 0 & \gamma_{6}
\end{array}\right) .
$$

The fact $\operatorname{det}\left(F_{1}^{\prime}(x, y, z)\right)=a$ nonzero contant implies that

$$
\operatorname{det}\left(\begin{array}{cc}
\left(\phi_{4}+L_{4}\right)_{x}^{\prime} & \left(\phi_{4}+L_{4}\right)_{y}^{\prime} \\
\left(\phi_{5}+L_{5}\right)_{x}^{\prime} & \left(\phi_{5}+L_{5}\right)_{y}^{\prime}
\end{array}\right)=a \text { nonzero constant. }
$$

Then the same arguments as in the proof of case (i) implies that $F_{1}$ is conjugate to one of the following maps:

$$
\begin{aligned}
H_{1}(x, y, z) & =(P(x, z)+a y, Q(z)+x, c z+d) \\
E(x, y, z) & =(P(y, z)+a x, Q(z)+b y, c z+d)
\end{aligned}
$$

where $P$ and $Q$ are polynomials with $\max \{\operatorname{deg}(P), \operatorname{deg}(Q)\}=2$ and $a b c \neq 0$.

We finish the proof of Theorem 2.1.

\section{Some dynamical properties of $H_{j}$}

Remark 4.1. For an elementary map $E(x, y, z)=(P(y, z)+a x, b y+$ $Q(z), c z+d), E$ has at most 1 isolated fixed point, in fact, it is easy to check the following facts:

(i) If $c \neq 1, a \neq 1, b \neq 1$, then $E$ has only one isolated fixed point;

(ii) If $c=1, d \neq 0$, then $E$ has no fixed point;

(iii) If $c=1, d=0$, then $E$ has a fixed $z$-plane for any given $z$ and the set of fixed points of map $E, \operatorname{Fix}(E)$, has only the following possibilities:

(1) Empty (for example: $b=1, Q(z)=1$ );

(2) Entire-curves;

(3) 2-dimensional complex surfaces. 
Remark 4.2. For the map $H_{1}(x, y, z)=(P(x, z)+a y, x+Q(z), c z+d)$, if $P(x, z)$ doesn't depend on $x$, then $H_{1}^{2}$ is a special case of our elementary map $E$. So the discussion of its dynamics goes to the study of elementary maps. If $c=1, d=0$ and $P(x, z)=k x^{2}+\cdots$ with $k \neq 0$, then this map is essentially an Hénon map of $\mathbb{C}^{2}$ for any fixed $z$. Therefore in general we believe that $H_{1}$ has interesting dynamics if $P(x, z)=k x^{2}+\cdots$ with $k \neq 0$.

Remark 4.3. For the map $\left.H_{2}(x, y, z)=(P y, z)+a x, z+Q(y), y\right)$, if $a=1$ and $P(y, z)=0$, then the map $H_{2}$ is essentially an Hénon map of $\mathbb{C}^{2}$ for any fixed $x$. So if $Q$ is a degree 2 polynomial, we believe that the map $\mathrm{H}_{2}$ has interesting dynamics. Otherwise we have some trivial examples like this: $H_{2}(x, y, z)=\left(y^{2}-x^{2}+x, y, z\right)$. It is easy to see that $H_{2}^{2}$ is an identity map.

Remark 4.4. For $H_{3}(x, y, z)=(P(x, z)+a y, z+Q(x), x)$, we have the following facts about its fixed points:

(i) If $P(x, x)+a Q(x)=0$, then we have 2 possibilities:

(i.a) If $a=1$, then $\operatorname{Fix}\left(H_{3}\right)$ is a entire curve;

(i.b) If $a \neq 1$, then $H_{3}$ has only one fixed point $(0, Q(0), 0)$.

(ii) If $P(x, x)+a Q(x) \neq 0$ and

(ii.a) The degree of the polynomial $P(x, x)+a Q(x) \leq 1$, then it is easy to see that the $\operatorname{Fix}\left(H_{3}\right)$ could be an empty set, one point or an entire curve;

(ii.b) The degree of the polynomial $P(x, x)+a Q(x)=2$, then it is easy to see that the $H_{3}$ has exactly 2 isolated fixed points counted with multiplicity. We believe that the $H_{1}$ in this case has rich dynamics.

Example 4.5. There are some interesting examples of $H_{3}$ in the case of $P(x, x)+a Q(x)=0$ and $a=1$ with $H_{3}^{3}$ is an identity map. For example, $H_{3}(x, y, z)=\left(z^{2}+y, z-x^{2}, x\right)$.

Remark 4.6. Follow the same discussion as in Remark 3.6, we know that $H_{4}(x, y, z)=(P(x, y)+a z, x+Q(y), y)$ has 2 isolated fixed points counted multiplicity if $P(y-Q(y), y)+Q(y)+(a-1) y$ is a degree 2 polynomial of $y$. We also believe that $H_{4}$ has rich dynamics in this case.

Remark 4.7. For the map $H_{5}(x, y, z)=(P(x, y)+a z, b y+Q(x), x)$, if $b=1$ and $Q(x)=0$, then the map $H_{5}$ is essentially an Hénon map of 
$\mathbb{C}^{2}$ for any fixed $y$. If $P$ and $Q$ are degree 2 polynomials, we believe that the map $H_{5}$ has interesting dynamics and different from the dynamics of Hénon map in $\mathbb{C}^{2}$.

There are also some uninteresting examples like this: $H_{3}(x, y, z)=$ $\left(2 x z, 2 y-x^{2}, x\right)$. It is easy to check this map has only one periodic point which is the fixed point at the origin.

Remark 4.8. For every map of $H_{j}$ and $E$, the degree of its inverse polynomial could be 3 or 4 if the $\operatorname{deg}(P)=\operatorname{deg}(Q)=2$. But it must be 2 if either $\operatorname{deg}(P) \leq 1$ or $\operatorname{deg}(Q) \leq 1$.

Remark 4.9. The detailed discussion of the dynamical properties of our maps $H_{j}$ and $E$ will appear in our forthcoming papers.

\title{
References
}

[F] S. Friedland, Two problems in complex dynamics, ?.

[FM] S. Friedland and J. Milnor, Dynamical properties of plane polynomial automorphisms, Ergodic Theory Dynam. Systems ? (1989), 67-99.

[HO] J. Hubbard and W. Oberste-Vorth, Hénon mappings in the complex domain II, IHES Publ. Math. 79 (1994), 5-46.

$[\mathrm{J}]$ H. W. E. Jung, Uber ganze birationale transformationes der Ebene, J. Reine Angew. Math. 184 (1942), 161-174.

\author{
Mathematics Department \\ The University of Michigan \\ 2072 EH, 525 E. University Ave. \\ Ann Arbor, MI 48109-1109 \\ U.S.A.
}

Rebut el 29 de maig de 1997 